\title{
Person Re-identification Based on Enriched Symmetry Salient Features and Graph Matching
}

\author{
Sara Iodice and Alfredo Petrosino \\ Department of Applied Science University of Naples Parthenope, Italy
}

\begin{abstract}
We propose a person re-identification non-learning based approach that uses symmetry principles, as well as structural relations among salient features. The idea comes from the consideration that local symmetries, at different scales, also enforced by texture features are potentially more invariant to large appearance changes than lower-level features such as SIFT, ASIFT. Finally, we formulate the re-identification problem as a graph matching problem, where each person is represented by a graph aimed not only at rejecting erroneous matches but also at selecting additional useful ones.

Experimental results on public dataset i-LIDS provide good performance compared to state-of-the-art results.
\end{abstract}

Keywords: Person Re-identification, Graph Matching, Symmetry.

\section{Introduction}

Symmetry detection is highly relevant in pattern recognition. Indeed, the description of a figure may be different when it is embedded in a context with horizontal or vertical symmetry [13. Besides, in tasks requiring the completion of partially occluded visual stimuli, subjects tend to produce systematically symmetrical figures [11. The concept of symmetry is not univocal: various kinds of properties of an image are defined as symmetry 24] 22. As instance, a figure has rotational symmetry when it can be rotated less than $360^{\circ}$ around its central point, or axis, and still matches the original figure.

This cue is peculiar in person re-identification where the problem consists in recognizing people in different poses from images coming from distinct cameras. This is an important task in the video surveillance, where large and structured environments must be supervised (such as airport, metro, station or shopping centers) and it becomes more critical when the cardinality of gallery set increases.

Like [5] we use symmetry principles, but not in a global exception, that is to say symmetry detected at high value of scale. In person re-identification context, global symmetry is not a good descriptor for pedestrian images, where there is often high variation of pose. On the contrary, we use local symmetry as descriptor like [2, based on the consideration that, at different scales, it is potentially more invariant to large appearance changes than lower-level features such as SIFT. Indeed, the symmetry feature combined with a texton-based feature is high discriminative [8]. 
According to the idea that an image can be described at the higher level in terms of a nested hierarchy of local symmetries, in this paper we present a novel graph matching approach to the problem aimed at evolving an initial set of correspondences computed with the local features, as a kind of compromise between the constraints imposed by both the local features and the structural relations. The vertices are the image key points detected by the SIFT (specifically we adopt ASIFT [21]), enriched by features based on color, texture, and local symmetry. The cost of an edge joining two vertices represents a measure of their dissimilarity. Therefore, the problem of Person Re-Identification is formulated as a graph matching problem. Our approach is an appearance-based method which differs from the state of art: $i$ ) unlike [3] [15] we give great weight to local features; $i i)$ we do not adopt spatio-temporal information such as [12] [10; iii) our method is non-learning based one, unlike [26] [1] [16].

The paper is organized as follows: the Sec. 2 deals with local features; in the Sec. 3 we provide details about how graphs are obtained from images and how they are compared. In Sec. 4 we show testing results on public dataset ILIDS and finally in Section 5, some conclusions are drawn.

\section{Feature Extraction}

First of all, we start with the phase of enhancement applying a Gaussian filter on the image. Then, we transform each image $I$ in the HSV space. In order to make the feature based on color invariant to lighting changes, we equalize the histogram of the $V$ component.

Later, for each pedestrian we detect image asymmetry axes. We obtain the axes of asymmetry combining opportunely two operators: chromatic bilateral operator $C$ and spatial covering operator $S$. Given two regions, the former $C$ calculates the color distance, the latter $S$ calculates the difference of FG (foreground). The asymmetry axes separate regions corresponding to human body's parts (head, trunk, and legs). The first axis, which is located at height $i T L$, separates regions with different chromatic contents but similar areas. These regions correspond to t-shirt/pants or suit/legs, for example. The second axis is calculated taking into account only the areas. This axis separates regions corresponding to head/body and is located at height $i H T$.

This step assumes that the background has been subtracted from each pedestrian image. We use for each image $I$ a mask $Z$ generated through the STEL model [7].

We have chosen to detect features separately because each region is characterized by contrasting aspects. Specifically, features based on color, symmetry, and texture are extracted. They can discriminate the trunk part from the legs part, as shown in Fig. 2] where the first three PCA components of feature vectors derived from the same region are plotted: the head components are red; the trunk components are green; the legs components are blue. It clearly appears 


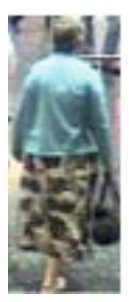

(a)

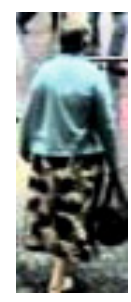

(b)

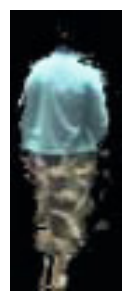

(c)

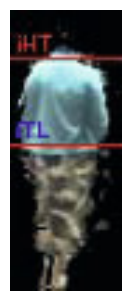

(d)

Fig. 1. Fig(a) Image 5003 in ILIDS dataset, Fig(b) Equalized Image, Fig(c) Background Subtraction, Fig(d) Asymmetry Axes at height $i H T$ and $i T L$

that blue and green components are concentrated in clusters. To resume, this experiment shows that the features are dicriminative; they can distinguish the trunk region from the legs region.

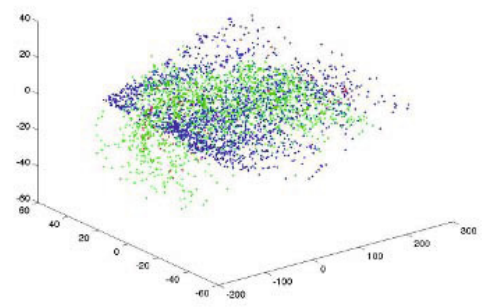

Fig. 2. First three PCs from head (in red), trunk (in green) and legs (in blue)

\subsection{Local and Multiscale Symmetry}

The property of being symmetrical finds correspondence in size, shape, and relative position of parts on opposite sides of a dividing line or median plane or about a center or axis. In particular, we deal with bilateral symmetry and the symmetry detection algorithm that has been considered in the present paper, named Kondra\&Petrosino algorithm, was one of the top winners of the "Symmetry Detection from Real World Images" competition at IEEE CVPR2011[17. We specifically take advantage of a measure obtained by using correlation with the flipped image around a particular axis. Indeed, in any direction, the optimal symmetry axis corresponds to the maximal correlation of a pattern with its symmetric version [4. 
Instead of taking every point in the image, we downsample original images to increase speed by filtering with circular steerable filters [19]. Also, reflecting the patch around both $x$-axis and $y$-axis will save half the rotations of the patch. For color images, the patch is reflected with respect to the three bands before doing the correlation.

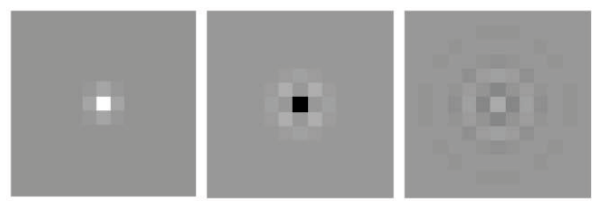

Fig. 3. Filters

\subsection{Texture}

Texture features are based on the technique proposed in [9]. Specifically, a twodimensional patch is "seen" for each interest point. The method uses 3 spatial filters of dimension $13 \times 13$ as shown in Figure 7 . The first is a Gaussian filter with $\sigma=0.5$, the second is a LoG filter with $\sigma=0.7$. Finally, the third filter is of the form

$$
F(r, \sigma, \tau)=\cos \left(\frac{\pi \tau r}{\sigma}\right) e^{\frac{r^{2}}{2 \sigma^{2}}}
$$

In this case $\sigma$ and $\tau$ are equal to 2. Each two-dimensional patch is convoluted with these filters, later thresholded so as to keep only the positive responses. The obtained binary image is denominated the "on" response. Thus, three binary images were obtained, and then one for each filter. The "on" responses are further convoluted with average filters, respectively of size $3 \times 3$ and $6 \times 6$ so as to obtain a multi-scale representation. At the next step, images are converted to grayscale and the intensity is normalized to have zero mean and variance equal to one. All filters have been normalized using a $L 1$ normalization, so that the responses of each filter are in the same range, i.e. each filter $F_{i}$ is divided by the $\left\|F_{i}\right\|$.

\section{From Person Re-identification to Graph Matching}

Each salient location detected by ASIFT [21] is enriched by the features described above. Each pedestrian image is rapresented by a graph, in order to consider structural relations. Furthermore, a graph can take into account the dissimilarity between keypoints. So the problem of person re-identification becomes a problem of graph matching. 


\subsection{Graph Construction}

Usually a Graph $G$ is a 3 -tuple $G=(V, E, Z)$ where $V$ is a set of vertices, $E \subseteq$ $V \times V$ is a set of edges, where $e \in E, e=\left(v_{i}, v_{j}\right)$ is an edge joining nodes $v_{i}, v_{j}$ $\in V$, and $Z$ is a set of vectors, where $z_{i} \in Z$ is the vector of attributes associated to node $v_{i} \in V$.

We build the graph for each pedestrian image as follows: vertices are the image key points detected by the ASIFT algorithm. We associate to each vertex a feature vector that contains features based on color $(R, G, B)$, texture, local symmetry and ASIFT descriptor $P_{i}=\left(X_{i}, R_{i}, U_{i}\right)$, where $X_{i}=\left(x_{i}, y_{i}\right)$ is its $2 D$ location, $R_{i}=\left(r_{i}, \alpha_{i}\right)$ its scale and orientation (in radians from $-\pi$ to $\pi$ ) and a vector of length 128 , i.e. $U_{i}=\left(U_{i, 1}, . ., U_{i, 128}\right)$, including information about the local texture on image.

The edge cost represents a measure of the joined vertex dissimilarity. We build an edge from vertex $v_{i}$ to vertex $v_{j}$ if $\operatorname{dist}\left(Z_{i}, Z_{j}\right)=\min \left(\operatorname{dist}\left(Z_{i}, Z_{k}\right)\right)$, $k=1, \ldots, m$ ( $m$ is the number of vertices) where dist is the Euclidean distance.

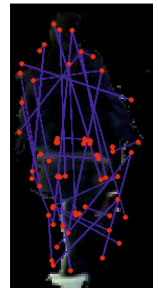

(a)

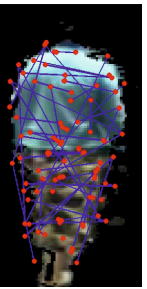

(b)

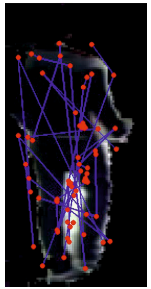

(c)

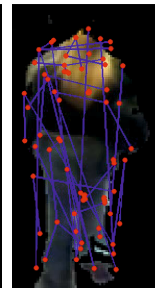

(d)

Fig. 4. Graphs of some ILIDS images: Fig(a) 3001; Fig(b) 5003; Fig(c) 13001 and Fig(d) 28005

\subsection{Graph Matching}

One main point is how to measure the contribution of matching one node to another with regards to the structural relations.

We adopt a revised version of the algorithm reported in [18. The distance between the vertices is obtained through the combination of two measures: the measure of similarity and the measure of consistency. The former is the Euclidean distance between the feature vectors; the latter takes into account the links between the vertices. The algorithm calculates the matching matrix. The matching matrix $S$ is a binary matrix defining an injective mapping between two graphs $G_{D}=\left(V_{D}, E_{D}, Z_{D},\right)$ and $G_{M}=\left(V_{M}, E_{M}, Z_{M},\right)$. An element $S_{i j} \in S$ is set to 1 if node $v_{i} \in V_{D}$ is matched to node $v_{j} \in V_{M}$ and 0 otherwise. A measure of structural consistency for the match $v_{i} \rightarrow v_{j}$ is given by: 


$$
Q_{i j}=\exp \left[\mu \sum_{k \in V_{d}} \sum_{l \in V_{m}} D_{i k} M_{j l} S_{k l}\right]
$$

where $\mu$ is a control parameter to avoid local minima. A cleaning heuristic is needed to obtain a binary matching matrix $S S$ that selects the matches corresponding to the highest coefficients according to a parameter $\rho$. We set the value of $\rho=0.8$ (to control the acceptance rate) and the valure of control parameter $\mu=0.15$. In fig. 4 we show the correspondences detected by the algorithm.

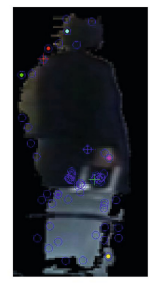

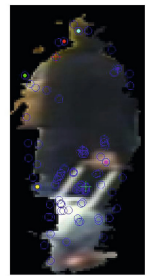

(a)
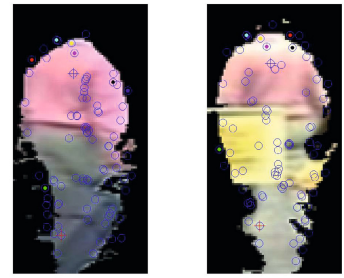

(b)

Fig. 5. Results achieved by the graph matching algorithm: Fig. (a) 0001001 vs 0001003 ; Fig. (b) 0002002 vs 0002004

\section{Validation and Testing}

We evaluate performance of our method on the i-LIDS MCTS dataset 20] depicting a real scenario observed by a multi-camera CCTV network monitoring the arrival hall of an airport. i-LIDS MCTS contains 479 images of variable size, for a total of 119 pedestrians. Each pedestrian is characterized at least by 2 images representing the same person wearing the same clothes. Such scenario is affected by several events such as illumination changes, occlusions, shape deformation and image blurring that make challenging the reidentification process.

\subsection{Experimental Setup}

We focus our attention on the challenging case MvsS, comparing our method with methods at state-of-the.art. [5] proposes SDALF, an appearence method that weights appearence informations extracted from different body parts, in agreement with their distance from symmetry axis calculated over the entire pedestrian image.

Zheng 25] tackles the MvsS case analyzing different types of visual features: Concatenated Histogram, Concatenated Histogram SIFT, and CRRRO-BRO.

In this setup, images are scaled to $128 x 64$ pixels and the probe set $P$ is built by selecting one image for each pedestrian randomly, while the gallery set $G$ is 
composed by the remaining images. Each image $p_{i} \in P$ is compared with each image $g_{i} \in G$ in order to evaluate the matching or score distance:

$$
\begin{aligned}
d\left(p_{i}, g_{j}\right)= & \alpha \cdot d_{W H}\left(W H\left(p_{i}\right), W H\left(s_{j}\right)\right)+ \\
& +\beta \cdot d_{G R A P H}\left(\operatorname{GRAPH}\left(p_{i}\right), \operatorname{GRAPH}\left(s_{j}\right)\right)
\end{aligned}
$$

where $W H(\cdot)$ (Weighted Color Histograms) [5] is the concatenated HSV histogram extracted by splitting the image $I$ into regions (head, trunk, legs) through horizontal asymmetry axes as described in sec. 2. $G R A P H(\cdot)$ is the graph representation of the pedestrian image as described in sec. 3.1 Vertices of the graph are characterized by concatenation of visual features (R, G, B, KIMA, TEXTONS1, TEXTONS2, ASIFT) and spatial information $(x, y)$, resulting in a final feature vector $F \in R^{138}$. Furthermore, KIMA is a measure of symmetry obtained by selecting maxima correlation between image patches $N \times N$ defined by flipping an image region over an axes having $M$ possible orientation and centered in (x,y). In our experiment we set $N=8$ and $M=12$. TEXTONS1 and TEXTONS2 are textural filters response evaluated in $(x, y)$ as described in sec. $2.2 d_{G R A P H}$ is the graph similarity distance described in sec. 3.1, $\alpha$ and $\beta$ are weighting values used to give different emphasis to $d_{W H}$ and $d_{G R A P H}$.

\subsection{Experimental Results}

Once all the matches have been evaluated, for each $p_{i} \in P$, we generate the ranking list composed by elements of $G$ in ascending order, whose first element rapresents the more similar pedestrian to $p_{i}$. In our experiment we obtain the best performances with these weighting values: $\alpha=0.4, \beta=0.6$. In fig. 6. CMC and SRR curves are reported. CMC [6] represents the re-identification rate of the system in function of rank position. Such measure is the analogous to the Roc Curve for detection problem [23. The SRR or SDR (Synthetic Disambiguation or Reacquisition Rate) converts a performance metric of size $N$ to one of size $M$, showing the probability that any of the $M$ best matches is correct [6]. SRR and $\mathrm{CMC}$ are related by:

$$
S D R(M)=S R R(M)=C M C(N / M)
$$

We repeat the experiment MvsS described above 100 times in order to build the CMC curve. As depicted in fig. 6, we achieve a percentage of $48 \%$ for rank 1 reidentification, producing results quite similiar to state-of-the-art method SDALF and outperforming Concatenated Histogram SIFT, Concatenated Histogram and CRRRO-BRO.

Such results are very promising since we are enriching ASIFT feature with a descriptor of simmetry that should be very discriminative also in condition of strong occlusions, where the SDALF method clearly should fail since it is based on global simmetry analysis. Unfortunally, the dataset we have utilized to evaluate re-identification methods, is not designed specifically on strong occlusions. State-of-the-art results are relative to the generic reidentification problem, where 
the number of occlusion is relatively low compared with the number of images. Our method works correctly on a strong occlusions, such as shown in figure 6] where it obtains rank 1 re-identification .

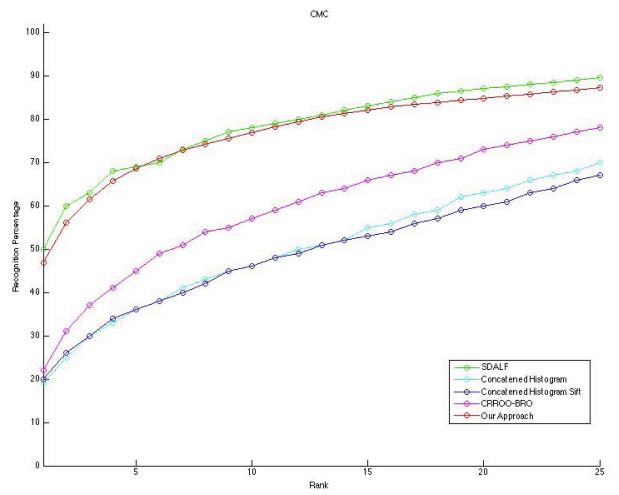

(a)

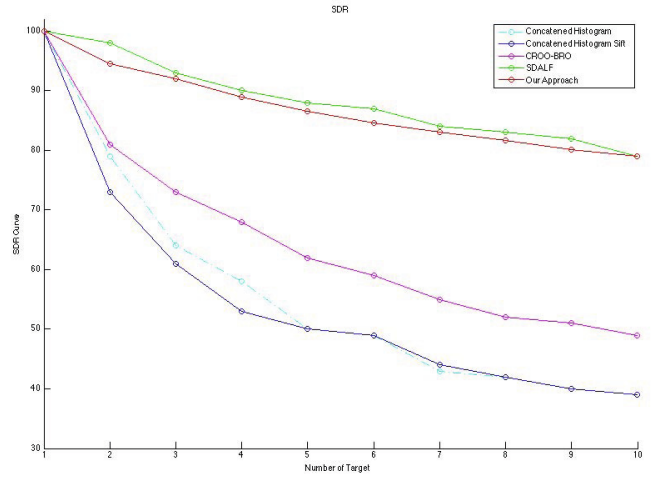

(b)

Fig. 6. Comparison in term of CMC (a) and SDR (b) on iLIDS MCTS dataset

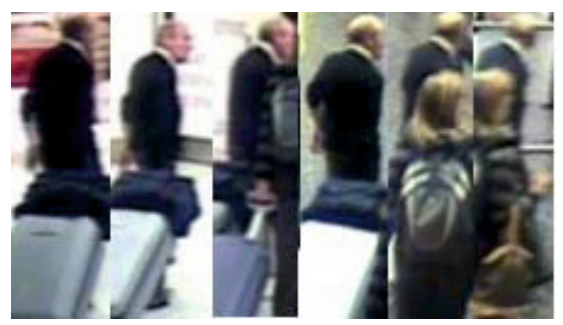

Fig. 7. Example of occlusion 


\section{Conclusions}

We report a new feature detector and descriptor based on ASIFT enriched by local symmetries detected densely across image and scale space, collected together in a graph representation. The basic features are designed for finding correspondence between difficult image pairs rich in symmetries, whilst the graph representation should be able to catch structural relations.

A graph matching method aimed at solving the point-set correspondence problem, takes into account relative structural and geometrical measurements.

The descriptor and results on the i-LIDS MCTS dataset demonstrates that the presence ASIFT feature enriched by local symmetries and structure of an individual is very informative for re-identification. The idea appears completely new, promising and opening a new perspective to the investigation of the occlusion problem, that should be furtherly analyzed. There is room for further improvements in accuracy, mainly for what concerns the tuning of parameter values and a sensitivity analysis.

\section{References}

1. Bauml, M., Bernardin, K., Fischer, M., Ekenel, H.K.: Multi-pose Face Recognition for Person Retrieval in Camera Networks. In: IEEE Int. Conf. on Advanced Video and Signal Based Surveillance (AVSS), Boston, MA, USA (2010)

2. Cabrini Hauagge, D., Snavely, N.: Image Matching using Local Symmetry Features. In: Proc. of IEEE CVPR 2012 (2012)

3. Cai, Y., Pietikäinen, M.: Person Re-identification Based on Global Context. In: Koch, R., Huang, F. (eds.) ACCV 2010 Workshops, Part I. LNCS, vol. 6468, pp. 205-215. Springer, Heidelberg (2011)

4. Di Gesú, V., Zavidovique, B.: The S-kernel: A Measure of Symmetry of Objects. Pattern Recognition 40(3), 839-852 (2007)

5. Farenzena, M., Bazzani, L., Perina, A., Murino, V., Cristani, M.: Person Reidentification by Symmetry-Driven Accumulation of Local Features. In: Proc. of IEEE CVPR 2010 (2010)

6. Gray, D., Brennan, S., Tao, H.: Evaluating Appearance Models for Recognition, Reacquisition, and Tracking. In: Gray, D., Brennan, S., Tao, H. (eds.) IEEE International Workshop on Performance Evaluation of Tracking and Surveillance, PETS (2007)

7. Jojic, N., Perina, A., Cristani, M., Murino, V., Frey, B.: Stel Component Analysis: Modelling spatial correlations in image class structure. In: Proc. of IEEE CVPR 2009 (2009)

8. Kondra, S., Petrosino, A.: Self-Similarity and Points of Interest in Textured Images. In: Kundu, M.K., Mitra, S., Mazumdar, D., Pal, S.K. (eds.) PerMIn 2012. LNCS, vol. 7143, pp. 306-313. Springer, Heidelberg (2012)

9. Kondra, S., Torre, V.: Texture Classification Using Three Circular Filters. In: 6th Indian Conference on Computer Vision, Graphics and Image Processing (ICVGIP), Bhubaneshwar, India (2008)

10. Kuo, C.-H., Huang, C., Nevatia, R.: Inter-Camera Association of Multi-Target Tracks by On-Line Learned Appearance Affinity Models. In: Daniilidis, K., Maragos, P., Paragios, N. (eds.) ECCV 2010, Part I. LNCS, vol. 6311, pp. 383-396. Springer, Heidelberg (2010) 
11. van Lier, R., Wagemans, J.S.: From Images to Objects: Global and Local Completion of Self-Occluded Parts. Journal of Experimental Psychology: Human Perception and Performance 25, 1721-1741 (1999)

12. Mazzon, R., Fahad Tahir, S., Cavallaro, A.: Person Re-identification in Crowd. Pattern Recognition Letters (2012) (in press)

13. Palmer, S.R.: The Role of Symmetry in Shape Perception. Acta Psychologica 59, 67-90 (1985)

14. Palmer, S.E., Hemenway, K.: Orientation and Symmetry: Effects of Multiple, Rotational, and Near Symmetries. J. Exp. Psychol. Hum. Percept. Perform. 4, 691-702 (1978)

15. Prosser, B., Gong, S., Xiang, T.: Multi-Camera Matching using Bi-directional Cumulative Brightness Transfer Functions. In: Proc. of the British Machine Vision Conf., Leeds, UK (2008)

16. Prosser, B., Zheng, W.S., Gong, S., Xiang, T.: Person Re-identification by Support Vector Ranking. In: Proc. of the British Machine Vision Conf., Aberystwyth, UK (2010)

17. Rauschert, I., Brocklehurst, K., Kashyap, S., Liu, J., Liu, Y.: First Symmetry Detection Competition: Summary and Results, CSE Dept Technical Report No. CSE11-012 (2011)

18. Sanroma, G., Alquzar Mancho, R., Serratosa i Casanelles, F.: A New Graph Matching Method for Point-Set Correspondence using the EM Algorithm and Softassig. Computer Vision and Image Understanding 116(2), 292-304 (2012)

19. Simoncelli, E.P., Freeman, W.T., Adelson, E.H., Heeger, D.J.: Shiftable Multi-Scale transforms. IEEE Trans Information Theory 38(2), 587-607 (1992)

20. U. H. Office : i-LIDS multiple camera tracking scenario definition (2008)

21. Yu, G., Morel, J.-M.: ASIFT: An Algorithm for Fully Affine Invariant Comparison, Image Processing On Line (2011), doi: http://dx.doi.org/10.5201/ipol.2011.my-asift

22. Wenderoth, P.: The Salience of Vertical Symmetry. Perception 23, 221-236 (1994)

23. Wilson, C., Hicklin, A.R., Bone, M., Korves, H., Grother, P., Ulery, B., Micheals, R., Zoep, M., Otto, S., Watson, C.: Fingerprint Vendor Technology Evaluation 2003: Summary of Results and Analysis Report. Technical Report NISTIR 7123, NIST (2004)

24. Zabrodsky, H.: Simmetry - A Review, Tech. Rep. 90-16, CS Dept., The Hebrew University of Jerusalem (1990)

25. Zheng, W.-S., Gong, S., Xiang, T.: Associating Groups of People. In: British Machine Vision Conference, BMVC (2009)

26. Zheng, W.S., Gong, S., Xiang, T.: Person Re-identification by probabilistic relative distance comparison. In: Proc. of IEEE Int. Conf. on Computer Vision and Pattern Recognition. Colorado Springs, CO (2011) 